\title{
Joint RANS/LES Modeling of Flameless Combustion
}

\author{
Vladimir L. Zimont, Valerio Battaglia \\ CRS4, Science and Technology Park Polaris, Pula, Italy \\ E-mail: zimont@crs4.it \\ Recieved May 26, 2011; revised July 15, 2011; accepted August 14, 2011
}

\begin{abstract}
We present our timesaving joint RANS/LES approach (we originally developed it for numerical simulations of turbulent premixed combustion) to simulate flameless combustion with separate injection of gas fuel and strong exhaust gas recirculation. It is based on successive RANS/LES numerical modeling where part of the information (stationary average fields) is achieved by RANS simulations and part (instantaneous nonstationary image of the process) by LES. The latter is performed using the RANS field of mean dissipation rate to model the sub-grid turbulent viscosity in the context of the Kolmogorov theory of small-scale turbulence. We analyze flameless combustion in the $\mathrm{FLOX}^{\circledR}$ combustor where we also simulate non-premixed flame combustion used for preliminary heating of the combustor. Different regimes take place using different systems of air injection. We applied for both regimes the simple assumption of "mixed is burnt". The main results are the following: 1) RANS simulations demonstrate for used two injection systems respectively more compact flame and distributed flameless combustion; 2) There is agreement between RANS and corresponding LES results: RANS and averaged LES profiles of the velocity and temperature are in reasonable agreement; 3) LES modeling with Kolmogorov independent on time sub-grid viscosity reproduce instantaneous image of the process including the vortex structures. Probably due to using an annular injector system for air the instantaneous field of the temperature demonstrate significant irregularity in the beginning of the burner, which in an animation looks like moving coherent structures; 4) In the joint RANS/LES approach the computer time of the LES sub-problems is much shorter than classic LES modeling due to using time independent subgrid transport coefficients and avoiding long-continued simulations, which are necessary for averageing of instantaneous LES fields. Practically in our simulations time consuming of the LES sub-problem was only several times lager then the RANS one and it makes this approach suitable for industrial applications.
\end{abstract}

Keywords: Flameless Combustion, Joint RANS/LES Modeling, FLOX Burner

\section{Introduction}

Combustion air preheating by flue gas heat recuperation and using of a particular regime of diluted combustion usually called in the literature "Flameless combustion" or "Flameless oxidation" is a promising technology for saving fuel, reduction of the $\mathrm{NO}_{\mathrm{x}}$ emissions and avoiding of unsteady combustion regimes [1-5].

Numerical simulations of the reacting flow inside an industrial burner is a fundamental tool for the development of new combustion systems that match the new emission limitations and the efficiency targets, the flameless combustion burner is one of the most promising system for industrial combustors. For practical applications the modeling approaches is still based mainly on the RANS formulation of the Navier-Stokes equations and the same situation takes place in the flameless com- bustion [1,6,7].

At the same time the RANS modeling presents some intrinsic limitations in the analysis of the flow characteristics as does not present the instantaneous nonstationary picture of the processes, the instantaneous structure of the turbulent eddies and reaction zones and so on. It is a reason why now in academic research there is a tendency to replace stationary RANS simulation by nonstationary LES tool. The latter has obvious fundamental advantage of avoiding modeling of the large-scale processes. At the same time LES arises fundamental and technical problems, which, in fact, renders replacing of RANS by LES difficult or even practically impossible for numerical simulations of industrial combustors with real geometry, injection systems and so on.

The aim of this work was to propose our joint RANS/LES approach for CFD simulations of the flame- 
less combustion. Originally this approach was aimed for premixed flames $[8,9]$ mainly applying to gas turbine clean combustion [10]. Here we modify this approach for simulation of the flameless combustion with separate injection of gas fuel and air. We try to suggest an economical tool for prediction of mean and instantaneous fields by combining positive properties of both RANS and LES approaches:

1) RANS is more economical in terms of computational resources and directly yields the required average fields on sufficiently refined meshes. In this sub-problem the large-scale turbulence is modeled statistically and we use in our simulation the standard " $\kappa-\varepsilon$ " turbulence model. For combustion modeling we assume infinitely fast chemistry and use a presumed PDF function for the passive concentration. Such approach is theoretically justified for a stabilized flame combustion as well as for stabile form of flameless oxidation (area $\mathrm{C}$ on the classical diagram of the stability limits for different combustion modes, Figure 9, [1]) and we analyze only this operating mode. A limit of this approach is that we cannot describe the unstable mode when takes place lift off and finally blow out of the flame when temperatures below self-ignition [1] (area B of the diagram) as well as the boundaries between the modes. In simulations we also ignore the effect of radiation.

2) In the LES sub-problem the large-scale structures of the flow and the reaction zone are directly resolved, and it is possible to analyze the subgrid smoothed instantaneous fields of the velocity, temperature, pressure and so on as well as the location of the reaction sheet. The main peculiarity of this approach is that LES modeling is based on a previous RANS simulation namely the subgrid turbulence in LES is estimated by the Kolmogorov theory of equilibrium small-scale turbulence using the mean dissipation rate $\varepsilon$ from the RANS simulation. In this case the subgrid viscosity has no pulsations and the time step can be significantly larger than at using traditional Smagorinsky model for subgrid turbulence.

It is significant that we do not need the time consuming procedure of averaging of the LES results as the mean fields follow directly from RANS simulations, and it the larger time step makes the LES modeling more friendly in comparison with known attempts to replace RANS simulation with LES. Nevertheless for methodological purpose we compare in this paper the RANS and averaged LES results to show the degree of agreement between RANS and LES results.

All numerical results refers to the FLOX ${ }^{\circledR}$ burner. $^{1}$ We simulate not only the flameless combustion regime of combustion but also the flame combustion one. The latter is used at initial stage for heating of the burner, which is necessary for realization of the flameless combustion.

${ }^{1} \mathrm{FLOX}^{\circledR}$ is a registered trademark by WS GmbH, Renningen.
Transition from the flame to the flameless combustion regime is performed by changing of the air injection inlet. The air is fed inside the burner through annular sections coaxial to the fuel port. Flame combustion is generated by an air inlet section around the fuel port. Shifting air inlet to a section at a radial distance of $4 \mathrm{~mm}$ from the fuel port, the inlet air mix with exhaust gas before mixing with the fuel, generating flameless combustion; additional details about this burner is given in a following section. Our simulation were performed in the context of the commercial code Fluent so the RANS sub-problem was simulated using the implemented equation for non-premixed combustion. Additional equations for the LES sub-problem where installed through the Fluent subroutines.

\section{The Basic Equations}

Here we shortly describes the main equations of the RANS and LES sub-problems and present data concerning the numerical procedure: used numerical methods, grids, computer system, time of simulations, used visualization method for animations, which illustrate LES results. We simulated the FLOX combustor with separate injection of fuel and air, i.e. in fact both flame and flameless combustion regimes correspond to different realizetions of non-premixed combustion with exhaust gas recirculation. We assume in our simulations equilibrium chemistry that is reasonable not only for stabilized flame, but also for stable flameless combustion [1]. The second simplification is that our modeling is based on using one mixture fraction $f$, which is a conservative scalar quantity that is characterized by instantaneous mixing.

The main idea of the joint RANS/LES approach is that we combine RANS and LES in a two-stage process. The first step consists of the RANS simulation which yields the averaged flow field; the second step entails LES using the dissipation $\tilde{\varepsilon}(x, t)$ obtained from RANS to estimate the subgrid turbulence. The latter staged gives a nonstationary image corresponding to the former stationary one.

\subsection{The RANS Sub-Problem}

The Favre average equations in terms of $\tilde{f}=\overline{\rho f} / \bar{\rho}$ and $\tilde{f}^{\prime \prime 2}=\overline{\rho f^{\prime \prime}} / \bar{\rho}\left(f^{\prime \prime}=f-\tilde{f}\right)$ are as follows:

$$
\begin{gathered}
\partial(\bar{\rho} \tilde{f}) / \partial t+\nabla \cdot(\bar{\rho} \tilde{u} \tilde{f})=\nabla \cdot\left(\left(\mu_{t} / \sigma_{t}\right) \nabla \tilde{f}\right) \\
\partial\left(\bar{\rho} \tilde{f}^{\prime \prime 2}\right) / \partial t+\nabla \cdot\left(\bar{\rho} \tilde{u} \tilde{f}^{\prime \prime 2}\right) \\
=\nabla \cdot\left(\left(\mu_{t} / \sigma_{t}\right) \nabla \tilde{f}\right)+C_{g} \mu_{t}(\nabla \tilde{f})^{2}-C_{d} \bar{\rho}(\tilde{\varepsilon} / \tilde{k}) \tilde{f}^{\prime \prime 2}
\end{gathered}
$$


with the following values of empirical constants:

$$
\sigma_{t}=0.85, C_{g}=2.86, C_{D}=2.0 \text {. }
$$

In simulations we used a presumed PDF $p(f)$ $\beta$-function. As a turbulence model we used the standard “ $\kappa-\varepsilon$ ” one presented in the Fluent code.

\subsection{The LES Sub-Problem}

As we mentioned above the main peculiarity of our LES modelling is using for estimation of the subgrid turbulence the Kolmogorov theory instead of traditional Smagorinsky model. Assuming the existence of Kolmogorov inertial spectrum $E(k)=C \varepsilon^{2 / 3} k^{-5 / 3}$ we can directly estimate the subgrid turbulent velocity and scale using $\varepsilon$ from a previous RANS simulation:

$$
\begin{aligned}
& u_{\Delta}^{\prime} \approx \int_{1 / \Delta}^{\infty} E(k) \mathrm{d} k \approx \varepsilon^{1 / 3} \Delta^{1 / 3}, \\
& L_{\Delta} \approx \int_{1 / \Delta}^{\infty} k^{-1} E(k) \mathrm{d} k / \int_{1 / \Delta}^{\infty} E(k) \mathrm{d} k \approx \Delta,
\end{aligned}
$$

and hence the subgrid turbulent transport coefficients are equal to

$$
\mu_{t}^{\Delta} \approx u_{\Delta}^{\prime} L_{\Delta} \approx \varepsilon^{1 / 3} \Delta^{1 / 3} .
$$

The field $\varepsilon(\vec{x})$ in Equation (4) was used from the RANS simulation so the sub-grid transport coefficient $\mu_{t}^{\Delta}$ depends only on coordinates $\mu_{t}^{\Delta}=f(x)$ and does not depends on $t$. This stationary $\mu_{t}^{\Delta}(x)$ is used in the non-stationary LES equation

$$
\begin{aligned}
& \partial\left(\bar{\rho}_{\Delta} \tilde{f}_{\Delta}\right) / \partial t+\nabla \cdot\left(\bar{\rho}_{\Delta} \tilde{u}_{\Delta} \tilde{f}_{\Delta}\right) \\
& =\nabla \cdot\left(\left(\mu_{t}^{\Delta} / \sigma_{t}\right) \nabla \tilde{f}_{\Delta}\right)
\end{aligned}
$$

The sub-grid Favre average parameters in Equation 5 also calculated using the PDF $\beta$-function where corresponding $\tilde{f}_{\Delta}^{\prime \prime 2}$ is estimated not from an sub-grid equation for the mixture fraction variance similar to Equation 2, but from an algebraic expression $\tilde{f}_{\Delta}^{\prime \prime 2}=C_{\mathrm{var}} L_{S}^{2}\left|\nabla \tilde{f}_{\Delta}\right|$, where $C_{\mathrm{var}}=0.5$ and $L_{S}=\Delta$. As we mentioned before, using of Equation 4 for estimation of the sub-grid viscosity instead of the Smagorinsky expression

$$
\mu_{t}^{\Delta}=\left(C_{S} \Delta\right)^{2}\left(2 \tilde{S}_{i j} \tilde{S}_{i j}\right)^{1 / 2} \quad\left(C_{S}=0.1\right) \quad \text { [11] strongly re- }
$$

duces the computer time. The reason is that the rateof-strain tensor for the resolved scale $\tilde{S}_{i j}$ has very strong fluctuations in space and time and in some points $(x, t) \mu_{t}^{\Delta}$ is very small so in nonstationary LES numerical modelling the time step $\Delta \tau$ must be significantly smaller than in the case of using Equation 4 as it is controlled by minimal values of the sub-grid viscosity. In accordance with our testing the computational time could be five times shorter than the time requested by the classic Smagorinsky model.

\section{The Burner Configuration}

We have simulated flame and flameless combustion in a model FLOX ${ }^{\circledR}$ burner aimed for steel treatments or glass, Figure 1. The fuel is natural gas and the nominal power $\mathrm{Q}_{\text {in }}$ is $13 \mathrm{~kW}$. The burner has an inner chamber with a radius of $0.02 \mathrm{~m}$ and a length of $0.41 \mathrm{~m}$; the exhaust gases flow in an outer coaxial tube with a diameter of $0.09 \mathrm{~m}$ and a length of $0.58 \mathrm{~m}$. The operative pressure is 1 bar. The exhaust recirculation take place through three windows in the inner tube. A sketch of the burner is showed in Figure 1. In the operative mode the burner is self-recuperative, the exhaust gases are used to preheat the inlet air; this aspects is not directly simulated and air inlet temperature is defined by previous experimental analysis and set to $980 \mathrm{~K}$. The injection system controls the operative combustion mode of the burner that can be switched from flame mode to flameless mode changing inlet air ports. In fact flameless combustion depends on the degree of exhaust gases recirculation that is controlled by the configuration of air inlet jets. The configuration that we tested has the characteristics presented in the Table 1, where $e_{\text {air }}$ is the excess of air and $k_{R}$ is the recirculation factor $k_{R}=m_{\text {exhast }} /\left(m_{\text {fuel }}+m_{\text {air }}\right)$.

\section{Results of Numerical Simulations}

Simulations were performed with the commercial code Fluent 6.2 on a cluster of 8 processors. The computational domain is a 3D 120 degree angular sector with periodic conditions at the lateral boundaries. Grid generation was made with Gambit and the final mesh has 1.5 $\mathrm{M}$ cells with hexahedral and tetrahedral elements.

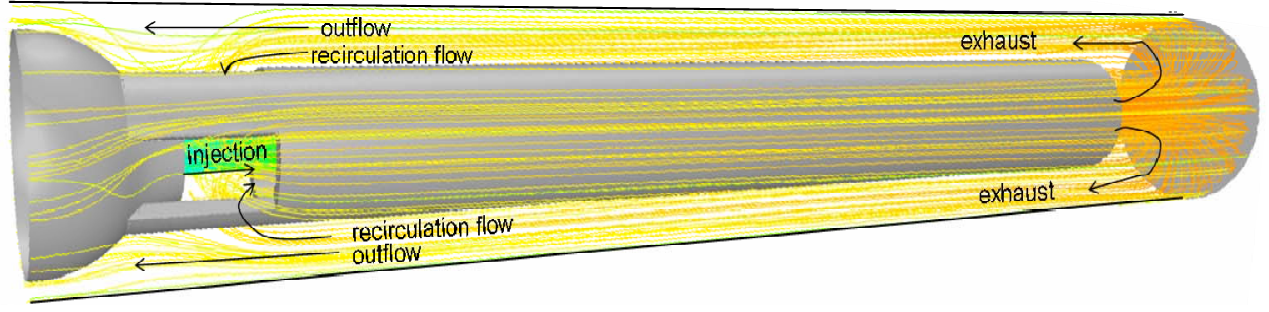

Figure 1. The sketch of the FLOX burner. 
Table 1. Burner operative parameters.

\begin{tabular}{ccccc}
\hline$Q_{\text {in }}[\mathrm{kW}]$ & $m_{\text {FUEL }}[\mathrm{kg} / \mathrm{s}]$ & $e_{\text {air }}[\%]$ & $A_{\text {air in }}\left[\mathrm{mm}^{2}\right]$ & $k_{R}[\%]$ \\
\hline 10.42 & $2.67 \mathrm{E}-04$ & 46 & 88 & 136 \\
\hline
\end{tabular}

Special refinement has been necessary near the inlet jets to resolve the strong velocity gradients in this region. As explained in the description of Joint RANS/LES approach, the simulation results consist of stationary RANS and unsteady LES.

\subsection{RANS Simulations of Flame and Flameless Combustion}

The turbulent model for RANS is the classic “ $\kappa-\varepsilon$ " turbulence model with standard wall functions. The convective scheme is second order upwind. The value of $k$ and $\varepsilon$ at the inlets derives by previous experimental analysis. The burner was simulated in flame and flameless conditions. Figure 2 shows the temperature field inside the burner for the both operative modes. It clearly demonstrates that the flameless mode presents very smooth temperature distribution compared to the flame mode that produces a hot spot in the first part of the inner tube. Figure 3 explains the reason of it: in the flame regime the isosurface with average stoichiometric composition is concentrated near the beginning of the burner while in the flameless regime this isosurface is more distributed along the chamber. Notice that relatively short averaged stoichiometric contour in the case of the flame mode is connected with large turbulent diffusion coefficient in this zone. At the same time dilution of air by products strongly increases the stoichiometric coefficient that results in very long averaged stoichiometrical contour. Figure 4 demonstrates axial that results in very long averaged stoichiometrical contour. Figure 4 demonstrates axial qualitative behavior of the axis temperature for both combustion regimes which corresponds to existing numerical simulations and experimental data presented in [1]. We notice that due to assumption of "mixed is burned" combustion in our simulations begins in both regimes directly in the section of injection of fuel and air. And only the intensity of combustion is different: it is much lower in the case of flameless combustion in comparison with the flame regime due to difference of the injection systems. At the same time optical measurements in [12] show that at the beginning of the burner there is no detectable emission of the $\mathrm{OH}$ radical so probably intensity of combustion in this zone is negligible that can be caused by influence of real chemical kinetics. We nevertheless think that the kinetic factor in the case of stable form of flameless combustion as well as for stabilized flame is not very significant in contrast to intermediate unstable combustion [1]. It is clear that boundary between these regimes as well as minimal furnace temperature, which is necessary for combustion, are controlled by both hydrodynamics and chemistry.

\subsection{Comparison of RANS and Averaged LES Numerical Results}

For the LES sub-problem a second order centered scheme was used for convective tem to reduce numerical diffusion. The turbulence at inlets was reproduced creating signals coherent with the velocity fluctuation and length scale given by experimental tests. The unsteady
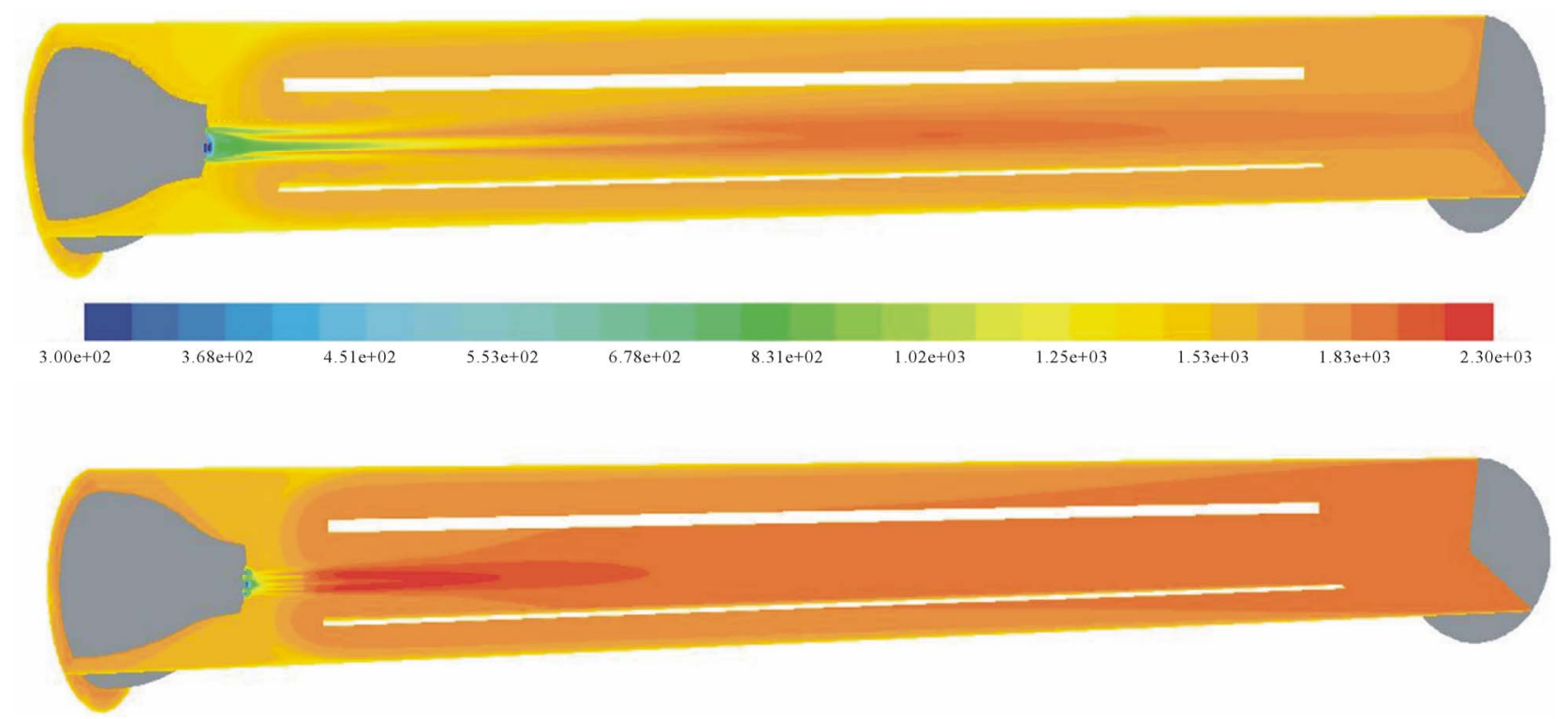

Figure 2. Flameless (top) and flame (bottom) operative mode. Field of Temperature (K). 


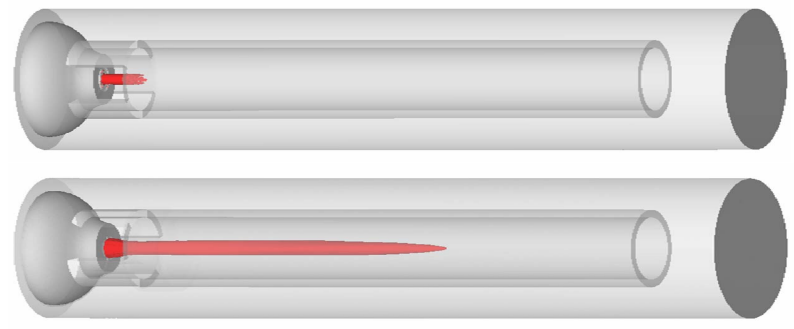

Figure 3. The stoichiometric isosurfaces.

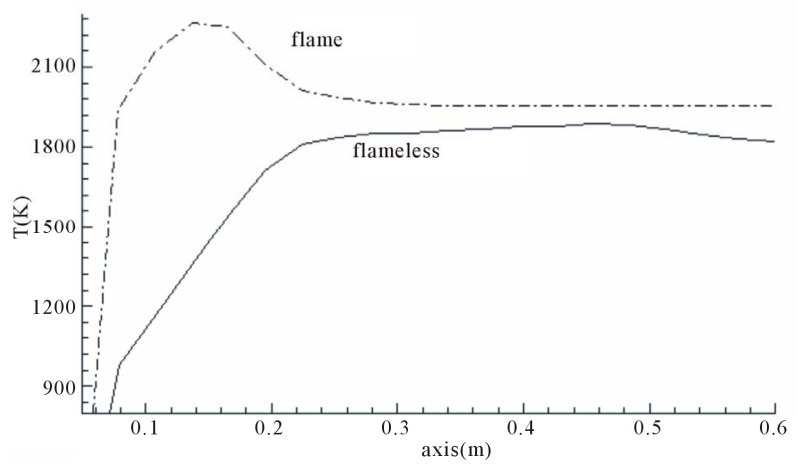

Figure 4. Qualitative distributions of the axial.

formulation was second order implicit in time. In LES sub-problem we concentrated in flameless mode to test LES in this particular conditions.

Key moment of the joint RANS/LES approach is correspondence of RANS and LES numerical results. We can check it by comparison of RANS and averaged LES results. For agreement between them, the turbulence models in RANS and LES sub-problems must be con- ceptually consistent as well as used in RANS simulations PDF function $p(f)$ must be similar to it following from LES in fact without modeling. Here we only notice that used in the LES sub-problem Kolmogorov theory of equilibrium small-scale turbulence [13] is also a basis of used in the RANS sub-problem “ $\kappa-\varepsilon$ ” turbulence model and the $\beta$-function is widely used as an acceptable approximation for $p(f)$.

Temperature fields in Figure 5 demonstrate qualitative agreement between RANS and averaged LES data while Figures 6 and 7, which present RANS and average LES profiles of the axial speed and temperature, show reasonable quantitative agreement between RANS and LES results.

It is necessary to stress that in practical applications of the joint RANS/LES approach time averaging of LES results that needs long-continued simulations is not necessary as the average fields follows directly from the RANS simulations. We presented the comparison of the RANS and average LES data only with a methodological aim: to show reasonable agreement between RANS and LES sub-problems.

\subsection{LES Picture of the Flameless Combustion}

LES data gives the opportunity to plot instantaneous fields of the flow. We have used Tecplot to postprocess LES data and produce the 2D and 3D pictures and also animations, which give vivid image of the process. Non-uniformity of the instantaneous field of the temperature at flameless combustion demonstrate Figures 8(a), (b) and (c) where instantaneous 3D configurations of the
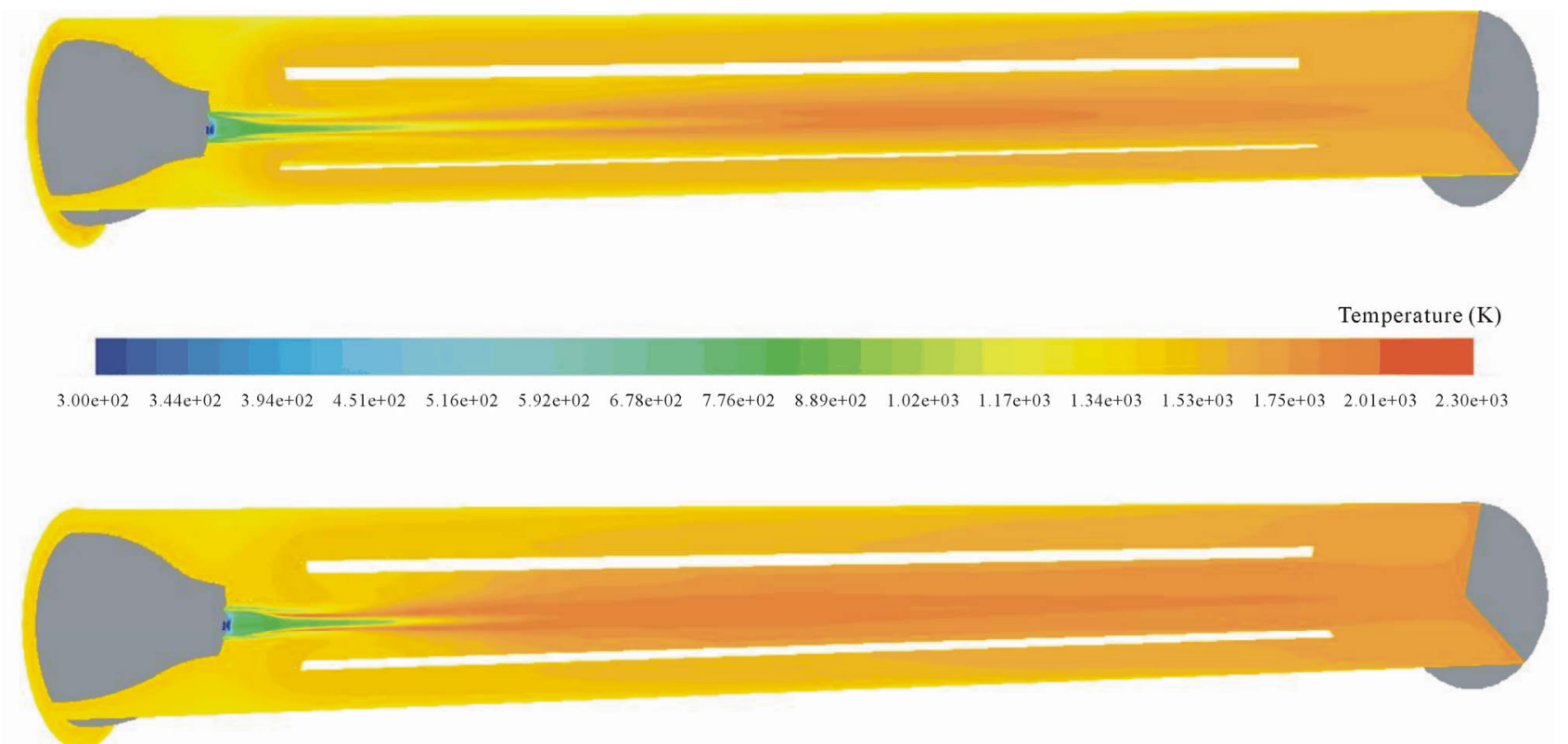

Figure 5. Top: RANS field of temperature. Bottom: averaged LES. 
$\begin{array}{llllll}\text { A B } & \text { C } & \text { D } & \text { E } & \text { F }\end{array}$

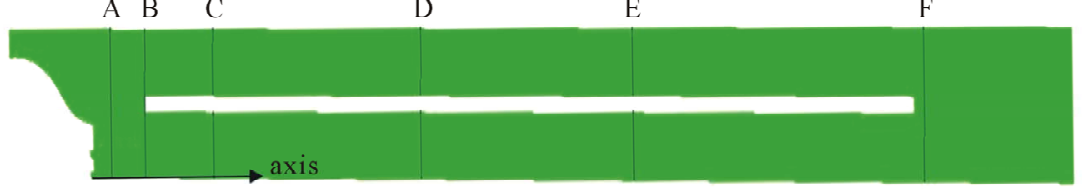
A: $\mathrm{x}_{\text {axis }}=0.01 \mathrm{~m}$
C: $\mathrm{x}_{\text {axis }}=0.07 \mathrm{~m}$
$\mathrm{E}: \mathrm{X}_{\mathrm{axis}}=0.31 \mathrm{~m}$
B: $\mathrm{x}_{\text {axis }}=0.03 \mathrm{~m}$
D: $\mathrm{x}_{\text {axis }}=0.19 \mathrm{~m}$
$\mathrm{F}: \mathrm{x}_{\mathrm{axis}}=0.51 \mathrm{~m}$
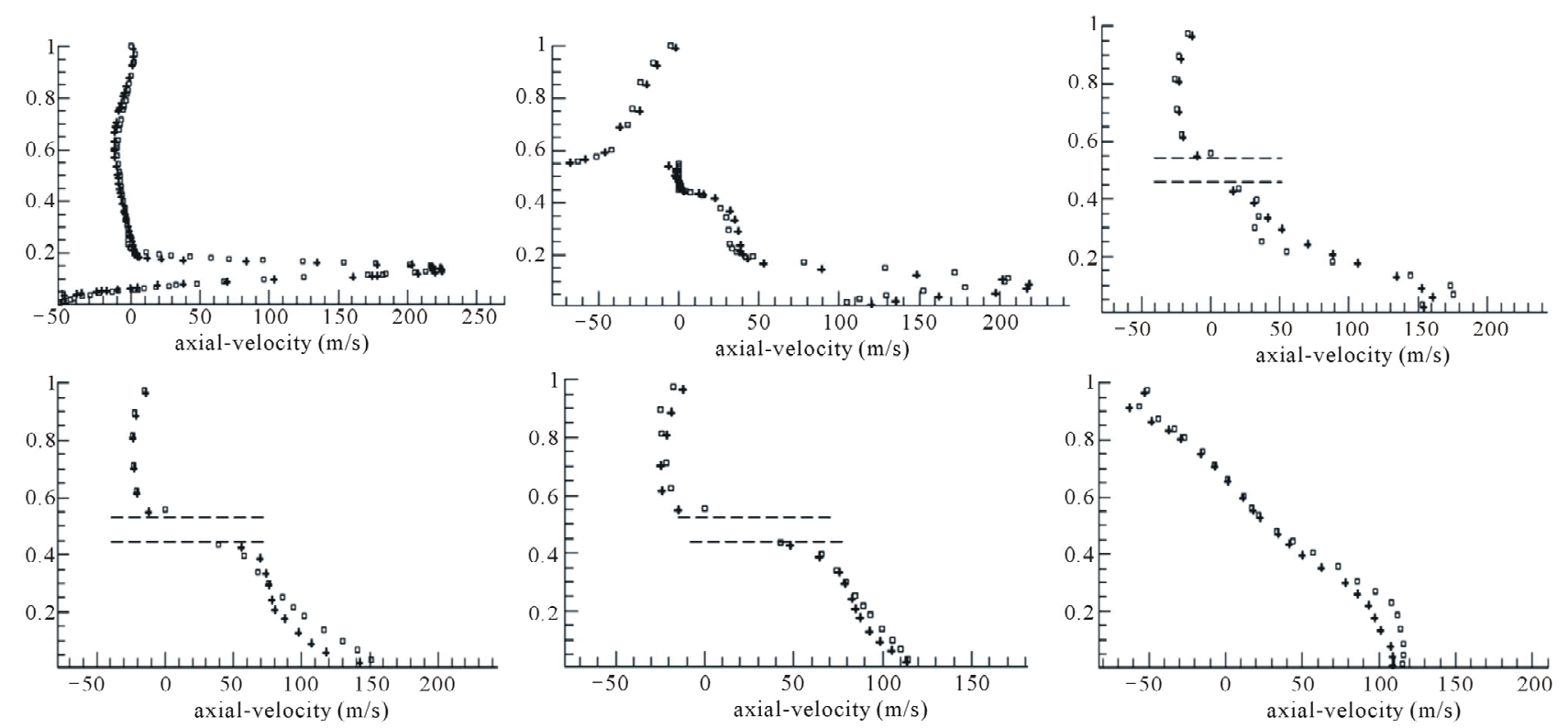

Figure 6. Comparison between RANS and averaged LES profile of axial-velocity. RANS: $\square$, LES: +.
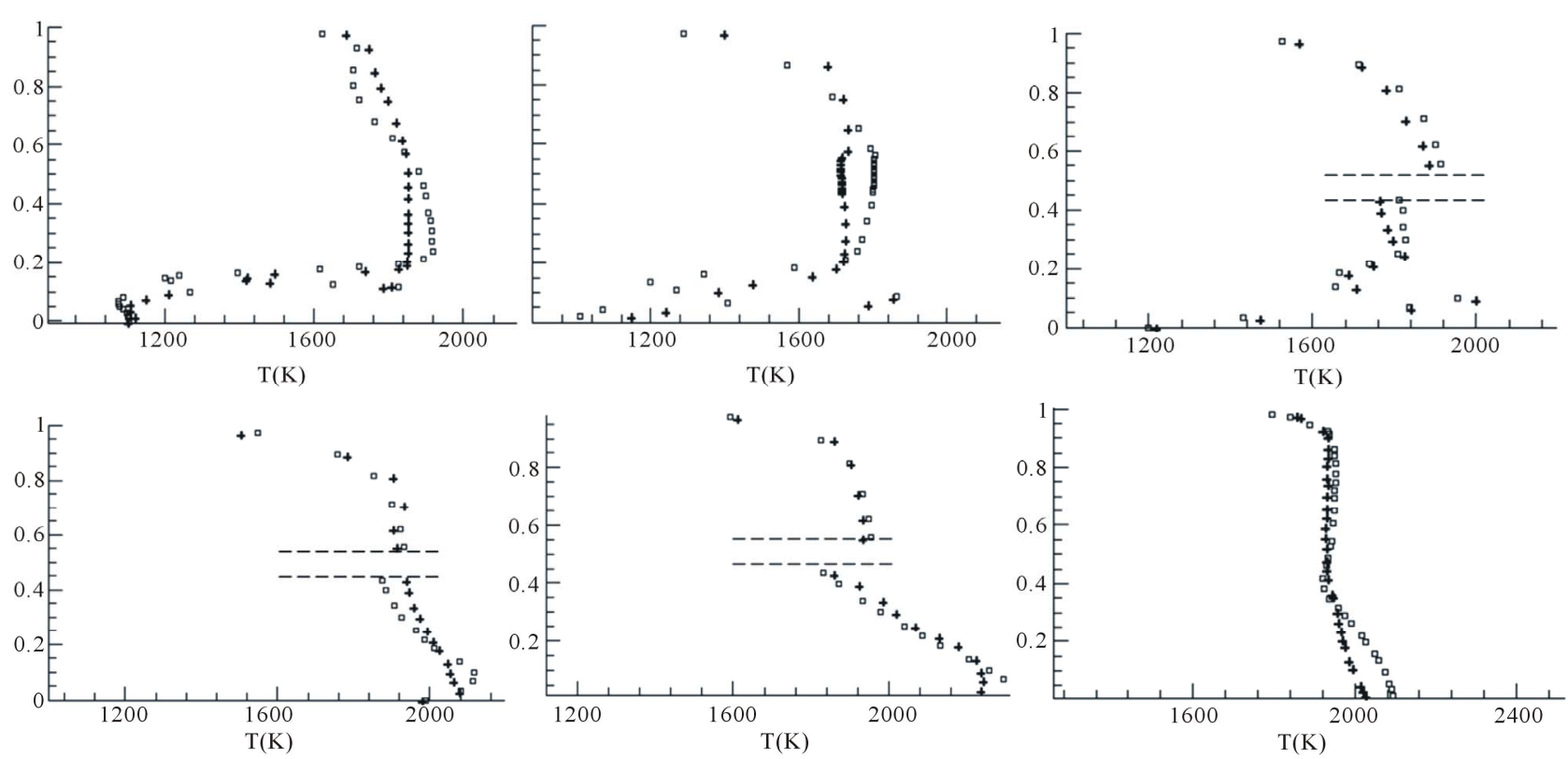

Figure 7. Comparison between RANS and averaged LES profile of temperature. RANS: $\square$, LES: + .

isotherms with the temperatures $\mathrm{T}=1700 \mathrm{~K}, 1900 \mathrm{~K}$ and $2100 \mathrm{~K}$ are presented: we see that gas with $\mathrm{T}=$ $1700 \mathrm{~K}$ concentrates near the wall in the back part of the burner. At the same time gas with $\mathrm{T}=1900 \mathrm{~K}$ is presented in all inner tube while the pots with $\mathrm{T}=2100 \mathrm{~K}$ mainly in the first part of the inner tube of the burner. 


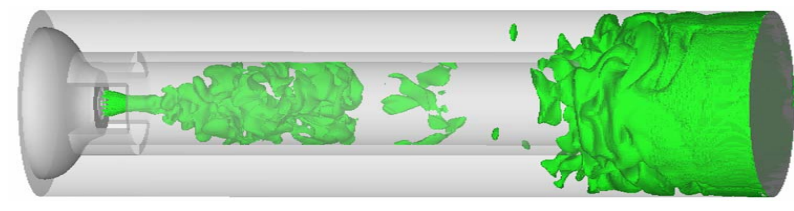

(a)

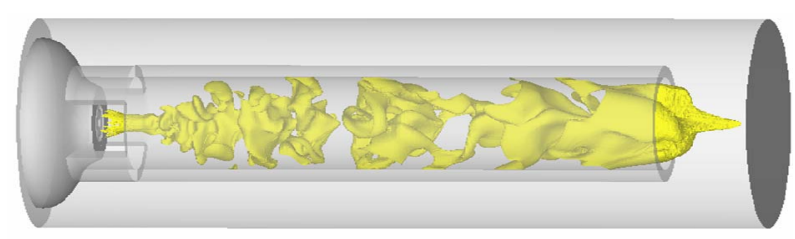

(b)

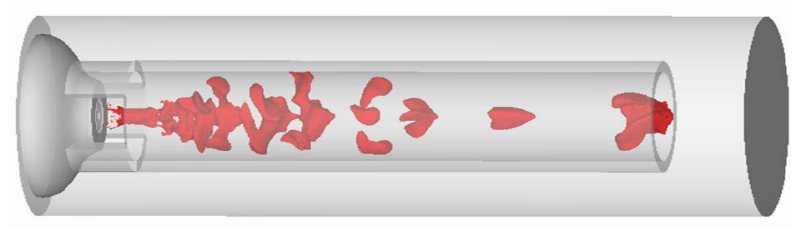

(c)

Figure 8. Instantaneous field of temperature. (a) $\mathrm{T}=1700 \mathrm{~K}$; (b) $\mathrm{T}=1900 \mathrm{~K}$; (c) $\mathrm{T}=2100 \mathrm{~K}$.
One instantaneous 2D image of simultaneous fields of temperature and pressure gradient from the animation is reproduced in Figure 9. We see from the field of the temperature that there is some kind of wrinkled sheet with higher temperature. The field of the pressure gradient demonstrates the vortexes structures. From the animations we clearly see that fluctuations in space and time of this sheet are controlled by turbulent eddies moving with the flow. 3D animation of the isotherm $\mathrm{T}=2200 \mathrm{~K}$ (one picture is shown in Figure 10 clearly demonstrates that this isotherm consists in separate pieces, which decrease in size and disappear moving along inner tube of the burner.

These instantaneous pictures demonstrate significant non-uniformity of instantaneous temperature. Known experiments also demonstrate large non-uniformity, for example, in an instantaneous profile of the temperature presented in [12] the difference between maximal and minimal temperature $\Delta \mathrm{T} \approx 600 \mathrm{~K}$. Instantaneous nonuniformity of the temperature field especially at first part of burner strongly depends on the used injector system. It is obvious that peculiarities of instantaneous non-uniformity of the temperature, which have limiting effect on

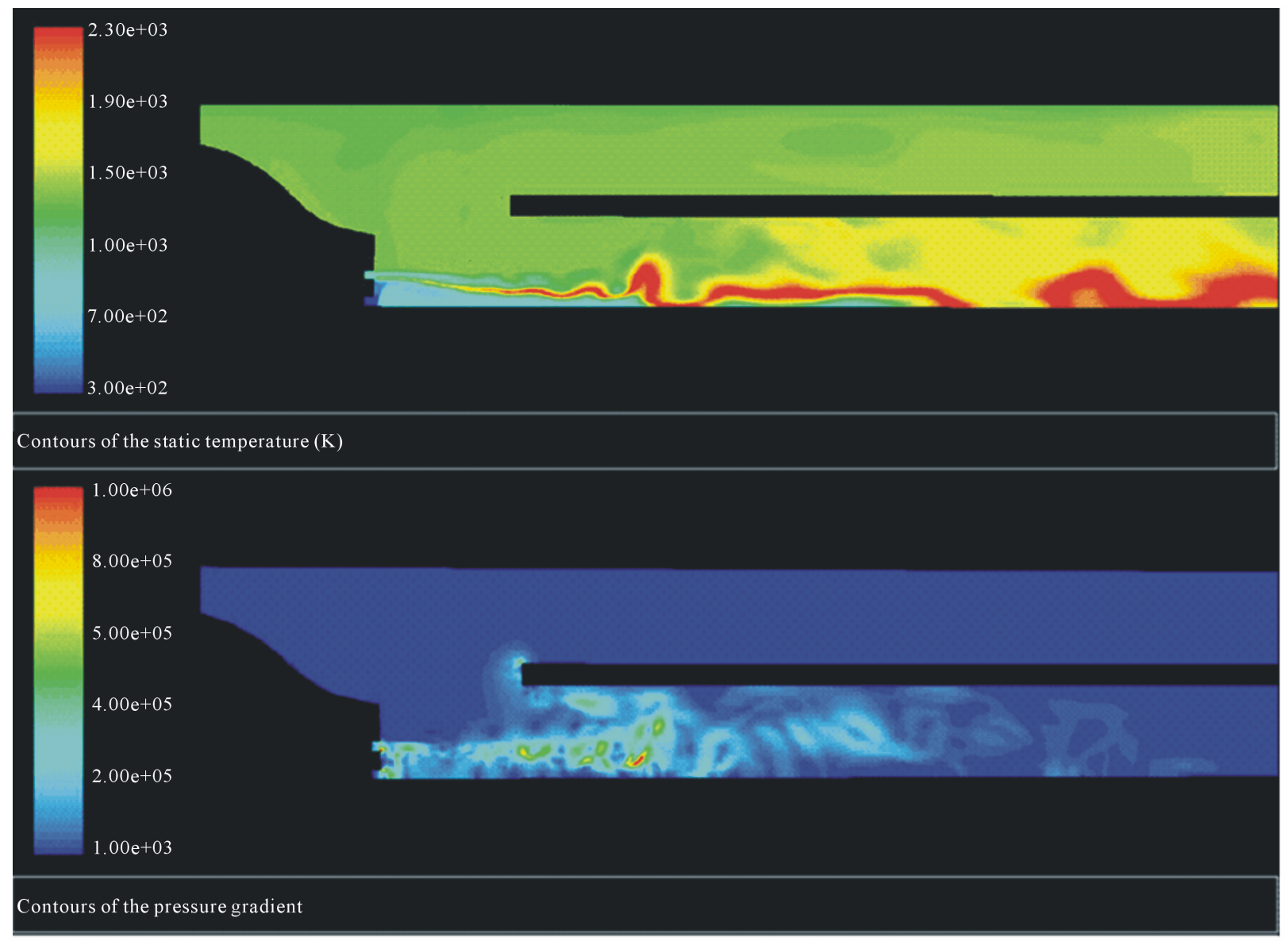

Figure 9. Instantaneous field of temperature and gradient pressure on a 2D section. 


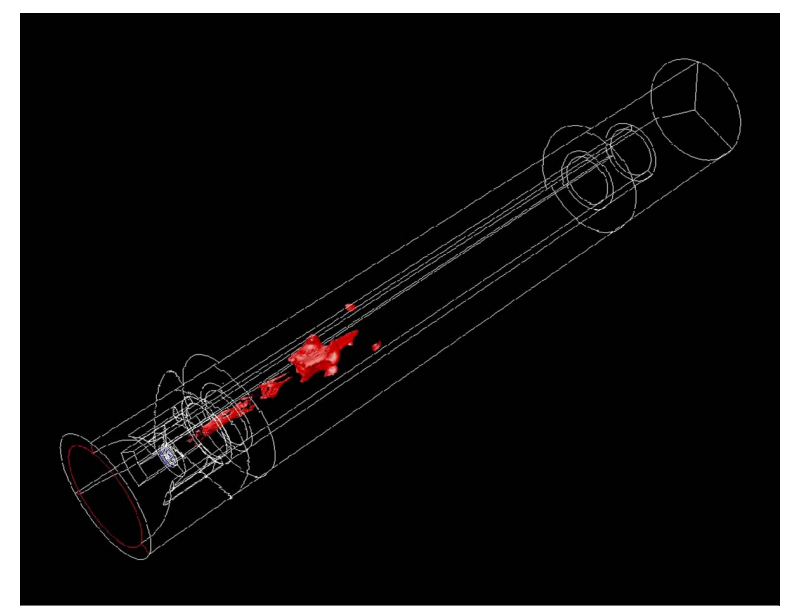

Figure 10. 3D instantaneous surface with the temperature $T$ $=2200 \mathrm{~K}$ from the $3 D$ animation.

the averaged fields, could strongly affect on $\mathrm{NO}_{\mathrm{x}}$ production. So optimization of the injector system in the context of the pollution problem is significant. We think that this optimization would be more effective using numerical data of the LES sub-problem. non-uniformity of the temperature field especially at first part of burner strongly depends on the used injector system. It is obvious that peculiarities of instantaneous non-uniformity of the temperature, which have limiting effect on the averaged fields, could strongly affect on $\mathrm{NO}_{\mathrm{x}}$ production. So optimization of the injector system in the context of the pollution problem is significant. We think that this optimization would be more effective using numerical data of the LES sub-problem.

\section{Conclusions}

1) We present the original timesaving joint RANS/LES approach to simulate flameless combustion with separate injection of gas fuel and strong exhaust gas recirculation. It is based on successive RANS and LES numerical modeling where some part of the information (stationary average fields) is achieved by RANS simulations and another part (instantaneous nonstationary image of the process) by LES. The latter is performed using the RANS field of the mean dissipation rate, which is used for modeling of the subgrid turbulence and subgrid viscosity in the context of the Kolmogorov theory of equilibrium small-scale turbulence.

2) Timesaving is achieved 1) due to use of the subgrid turbulent transport coefficient from the Kolmogorov theory of small-scale turbulence instead of the traditionally used Smagorinsky model (and it results in the possibility to increase the time step in our LES approximately five times) and 2) due to avoiding time averaging of LES data, which need long-continued simulations, which are ine- vitable in an approach "LES instead of RANS". In our RANS/LES approach ratio of necessary times for RANS and LES sub-problems in practical application could be the same order of magnitude (practically $\sim 1$ - 5).

3) For validation of our approach we performed longcontinued large eddy simulations. Presented time averaged LES data and RANS results are in reasonable agreement.

4) Though RANS results for the flameless regime demonstrate distributed combustion with smooth profiles of the temperature and velocity, corresponding instantaneous LES fields show significant nonuniformity of the temperature and clear documented large-scale eddies especially at initial part of the burner. As these temperature surges can be significant for $\mathrm{NO}_{\mathrm{x}}$ emission the burner and especially the injection system need optimization. We think that using for this optimization the results of the LES sub-problem could be more effective in comparison with using of the RANS one.

\section{References}

[1] J. A. Wünning and J. G. Wünning, "Flamneless Oxidation to Reduce Thermal NO-Formation," Progress in Energy and Combustion Science, Vol. 23, No. 1, 1997, pp. 81-94. doi:10.1016/S0360-1285(97)00006-3

[2] A. Cavaliere and M. De Joannon, "Mild Combustion," Progress in Energy and Combustion Science, Vol. 30, No. 4, 2004, pp. 329-366. doi:10.1016/j.pecs.2004.02.003

[3] T. Hasegawa and R. Tanaka, "High Temperature Air Combustion: Revolution in Combustion Technology,” JSME International Journal, Series B, Vol. 40, No. 4, 1998, pp. 1079-1084

[4] J. G. Wünning, “FLOX ${ }^{\circledR}$-Flameless Combustion,” Thermprocess Symposium VDMA, Düsseldorf, 2003. http://www.flox.com/de_documents/Paper64\%20tp03.pdf

[5] J. G. Wünning, "Flameless Combustion and its Applications,” 14th IFRF Members Conference, Noordwijkerhout, 2004. http://www.flox.com/de_documents/pra00045.pdf

[6] P. J. Coelho and N. Peters, "Numerical Simulation of a Mild Combustion Burner," Combustion and Flame, Vol. 124, No. 3, 2001, pp. 503-518. doi:10.1016/S0010-2180(00)00206-6

[7] S. Murer, B. Perenti and P. Lybaert, "Simulation of Flameless Combustion of Natural Gas in a Laboratory Scale Furnace," Turkish Journal of Engineering \& Environmental Sciences, Vol. 30, No. 3, 2006, pp. 135-143.

[8] V. Zimont and V. Battaglia, “Joint RANS/LES Numrical Simulations of Premixed Combustion at Strong Tuibulence," Ninth International Conference on Numerical Combustion (SIAM), Sorrento, 7-10 April 2002, Paper No. 141, pp. 225-226.

[9] V. L. Zimont and V. Battaglia, "Joint RANS/LES Approach to Premixed Flame Modelling in the Context of the TFC Combustion Model," Flow, Turbulence and Combustion, Vol. 77, No. 1-4, 2006, pp. 305-331. 
[10] V. L. Zimont, V. Moreau, V. Battaglia and R. Modi, "RANS and LES Modelling of the GE10 Combustor," In: Biblioneca Termotechnica (Proceedings of the ASME ATI Conference on "Energy: Production, Distribution and Conservation”, Milan, 14-17 May 2006, Vol. 2, No. 34, 2006, pp. 923-932.

[11] J. Smagorinsky, "General Circulation Experiments with the Primitive Equations I. The Basic Experiment," Monthly Weather Review, Vol. 91, No. 3, 1963, pp. 99-164. doi:10.1175/1520-0493(1963)091<0099:GCEWTP>2.3.CO;2
[12] T. Plessing, N. Peters and G. Wunning, "Laseroptical Investigation of Highly Preheated Combustion with Strong Exhaust Gas Recirculation,” Symposium (International) on Combustion, Vol. 27, No. 2, 1998, pp. 3197-3204.

[13] A. N. Kolmogorov and A. N. Doklady, "The Local Structure of Turbulence in Incompressible Viscous Fluid for Very Large Reynolds Number,” Doklady AN SSSR, Vol. 30, No. 4, 1941, pp. 299-303. (Reprinted in Proceedings of the Royal Society of London. Series A, Vol. 434, 1991, pp. 9-13). 